\title{
The Effect of Psychological Factors on Sleep Disturbances in Young Adults
}

Hye Sook Park

Department of Dental Technology, Shingu College, Seongnam, Korea

Received February 11, 2020

Revised March 15, 2020

Accepted March 16, 2020
Correspondence to:

Hye Sook Park

Department of Dental Technology of

Shingu College, 377 Gwangmyeong-ro,

Seongnam13174, Korea

Tel: +82-31-740-1575

Fax: +82-31-740-1589

E-mail: hspark@shingu.ac.kr

https://orcid.org/0000-0002-2981-3908

This study was supported by a grant from Shingu College.
Purpose: The purpose of this study was to assess influences of psychological factors on sleep disturbances in young adults through the revised version of the Minnesota Multiphasic Personality Inventory (MMPI-2).

Methods: Two hundred and ninety nine college students in Gyeonggi-do completed the MMPI-2 and a questionnaire related to sleep disturbances and collected data were analyzed by logistic regression.

Results: The odds of tooth grinding increased significantly with the increase of T-score of hysteria (Hy) scale. The increase of T-score of hypomania (Ma) and social introversion (Si) scale significantly contributed to the risk of insomnia. The occurrence of insomnia increased significantly as T-score of somatic complaints (RC1) and psychoticism (PSYC) scale increased. The odds of tooth grinding increased significantly as T-score of anxiety (ANX) and family problems (FAM) scale increased. The occurrence of insomnia decreased significantly with the increase of T-score of type A behavior (TPA) scale. The increase of T-score of dominance (Do) scale significantly contributed to the risk of tooth clenching. The odds of tooth grinding decreased significantly as T-score of MacAndrew alcoholism-revised (MACR) scale increased, whereas it increased significantly as T-score of addiction potential scale (APS) increased. No scale of MMPI-2 significantly affected the occurrence of snoring.

Conclusions: T-scores of Hy, ANX, FAM, Do, MAC-R, and APS scales on MMPI-2 affected the risk of bruxism. T-scores of Ma, Si, RC1, PSYC, and TPA scales on MMPI-2 influenced the occurrence of insomnia. The psychometric instrument such as MMPI-2 is helpful in understanding and managing bruxism and insomnia.

Key Words: Bruxism; Insomnia; Minnesota Multiphasic Personality Inventory-2; Sleep disturbances

\section{INTRODUCTION}

As a person's life style becomes more complicated, the occurrence of sleep disturbances is increasing [1]. The most common sleep disturbances comprise bruxism, snoring and insomnia. Bruxism includes both tooth clenching which is a continuously static position of maximum intercuspation and tooth grinding indicating a forceful movement of the jaw from side to side [2]. Snoring can be associated with obstructive sleep apnea characterized by intermittent collapse of the upper airway during sleep. The prevalence rate of snoring was reported $25.7 \%$ in young adults [3]. Insomnia usually shows the delay of sleep onset, difficulty staying asleep, or awakening too early, which may contribute to initiating or perpetuating orofacial pain [4].

There have been reports on the association of psychological status with sleep disturbances [5-7]. Kang et al. [1] showed the close relation between sleep quality and

Copyright (C) 2020 Korean Academy of Orofacial Pain and Oral Medicine. All rights reserved

(c) This is an open-access article distributed under the terms of the Creative Commons Attribution Non-Commercial License (http://creativecommons.org/licenses/by-nc/4.0/), which permits unrestricted non-commercial use, distribution, and reproduction in any medium, provided the original work is properly cited. 
psychogenic condition using the Symptom Checklist-90Revised (SCL-90-R) and Pittsburgh Sleep Quality Index. Manfredini et al. [8] supported an association between bruxism and certain psychopathological symptoms by means of self-report version of the Structured Clinical Interview for Panic-Agoraphobic Spectrum. Restrepo et al. [9] found a strong correlation among bruxism, the high anxiety level and the high tension personality trait using the Children's Personality Questionnaire and the Conners' Parents Rating Scales. Enright et al. [10] reported that observed apneas during sleep were associated with depression in elderly women through the modified Center for Epidemiologic Studies Depression. Buysse et al. [11] confirmed the increased risk of subsequent depression among individuals with insomnia by means of SCL-90-R.

The revised version of the Minnesota Multiphasic Personality Inventory (MMPI-2) is one of the most widely used psychometric instruments. Higher scores in MMPI-2 indicating maladaptive personality traits predicted incident chronic insomnia [12]. The psychological characteristics of the patients with bruxism were asessed by the Minnesota Multiphase Personality Inventory (MMPI) [13]. The associations between polysomnographic variables in obstructive sleep apnea and a variety of psychological responses including depressive symptoms were evaluated by MMPI [14]. Though these studies have been performed for various age groups, the study in young adults is rare. Hence, the aim of current study is to assess influences of psychological factors on sleep disturbances through MMPI-2 in young adults.

\section{MATERIALS AND METHODS}

This study is approved by the Institutional Review Board of Shingu College (IRB-2019-101).

\section{Subjects}

Data were collected from two hundred and ninety nine college students in Gyeonggi-do, including seventy six men and two hundred and twenty three women. Mean age of the subjects was 19.2 \pm 2.7 years (Table 1 ).

Data were obtained from October to December of 2019. Visiting a classroom, an examiner explained each item of the questionnaire (Appendix 1) related to sleep disturbances and asked the students to answer it by self-evaluation and retrieved the completed answers at their classroom. Moreover, a psychologist administered MMPI-2 to the students.

\section{Psychological Evaluation}

The MMPI-2 scales consist of the clinical scales, restructured clinical $(\mathrm{RC})$ and personality psychopathology five (PSY-5) scales, the content scales, and supplementary scales. Every clinical scale concerns a particular psychological aspect:hypochondriasis (Hs), depression (D), hysteria (Hy), psychopathic deviation (Pd), masculinity-femininity (Mf), paranoia (Pa), psychasthenia (Pt), schizophrenia (Sc), hypomania (Ma), social introversion (Si). A set of RC scales are less intercorrelated and have greater discriminant validity than the original clinical scales:demoralization $(\mathrm{RCd})$, somatic complaints ( $\mathrm{RC} 1$ ), lowpositive emotions ( $\mathrm{RC} 2$ ), cynicism (RC3), antisocial behavior (RC4), ideas of persecution (RC6), dysfunctional negative emotions (RC7), aberrant experiences (RC8), hypomanic activation (RC9). The PSY-5 scales were constructed to assess personality traits relevant to both normal functioning and clinical problems: aggressiveness (AGGR), psychoticism (PSYC), disconstraint (DISC), negative emotionality/neuroticism (NEGE), introversion/low positive emotionality (INTR). The content scales represent well the content dimensions of the original MMPI: anxiety (ANX), fears (FRS), obsessiveness (OBS), depression (DEP), health concerns (HEA), bizarre mentation (BIZ), anger (ANG), cynicism (CYN), antisocial practices (ASP), type A behavior (TPA), low self-esteem (LSE), social discomfort (SOD), family problems (FAM), work interference (WRK), negative treatment indicators (TRT). The supplementary scales are judged to be a very helpful supplementary source of information in interpreting the clinical scales: anxiety (A), repression $(\mathrm{R})$, ego strength (Es), dominance (Do), social responsibility (Re), college maladjustment (Mt), post-traumatic stress

Table 1. Demographics of the subjects

\begin{tabular}{ccc}
\hline Gender & Subject & Age $(\mathrm{y})$ \\
\hline Men & $76(25.4)$ & $20.1 \pm 3.9$ \\
Women & $223(74.6)$ & $18.9 \pm 2.1$ \\
Total & $299(100.0)$ & $19.2 \pm 2.7$ \\
\hline
\end{tabular}

Values are presented as number (\%) or mean \pm standard deviation. 
Table 2. Logistic regression analysis of clinical scales on sleep disturbances $(n=299)$

\begin{tabular}{|c|c|c|c|c|c|c|}
\hline \multirow{2}{*}{ Clinical scales } & \multirow{2}{*}{$\beta$} & \multirow{2}{*}{ SE } & \multirow{2}{*}{$\mathrm{p}$-value } & \multirow{2}{*}{ OR } & \multicolumn{2}{|c|}{$95 \% \mathrm{Cl}$} \\
\hline & & & & & Lower limit & Upper limit \\
\hline \multicolumn{7}{|l|}{$\mathrm{Hs}$} \\
\hline Tooth clenching & 0.002 & 0.036 & 0.959 & 1.002 & 0.933 & 1.075 \\
\hline Tooth grinding & -0.057 & 0.039 & 0.151 & 0.945 & 0.875 & 1.021 \\
\hline Snoring & 0.038 & 0.031 & 0.216 & 1.039 & 0.978 & 1.105 \\
\hline Insomnia & 0.065 & 0.034 & 0.052 & 1.068 & 0.999 & 1.140 \\
\hline \multicolumn{7}{|l|}{ D } \\
\hline Tooth clenching & -0.011 & 0.025 & 0.663 & 0.989 & 0.942 & 1.039 \\
\hline Tooth grinding & 0.005 & 0.025 & 0.849 & 1.005 & 0.956 & 1.056 \\
\hline Snoring & -0.017 & 0.021 & 0.425 & 0.983 & 0.943 & 1.025 \\
\hline Insomnia & 0.028 & 0.022 & 0.215 & 1.028 & 0.984 & 1.075 \\
\hline \multicolumn{7}{|l|}{ Hy } \\
\hline Tooth clenching & 0.044 & 0.036 & 0.224 & 1.045 & 0.973 & 1.122 \\
\hline Tooth grinding & 0.095 & 0.039 & $0.016^{*}$ & 1.099 & 1.018 & 1.187 \\
\hline Snoring & -0.026 & 0.032 & 0.416 & 0.975 & 0.916 & 1.037 \\
\hline Insomnia & -0.020 & 0.033 & 0.537 & 0.980 & 0.918 & 1.045 \\
\hline \multicolumn{7}{|l|}{$\mathrm{Pd}$} \\
\hline Tooth clenching & -0.036 & 0.025 & 0.150 & 0.965 & 0.919 & 1.013 \\
\hline Tooth grinding & -0.046 & 0.026 & 0.074 & 0.955 & 0.908 & 1.005 \\
\hline Snoring & -0.003 & 0.020 & 0.885 & 0.997 & 0.958 & 1.037 \\
\hline Insomnia & 0.009 & 0.021 & 0.670 & 1.009 & 0.968 & 1.052 \\
\hline \multicolumn{7}{|l|}{ Mf } \\
\hline Tooth clenching & -0.005 & 0.014 & 0.742 & 0.995 & 0.968 & 1.023 \\
\hline Tooth grinding & 0 & 0.015 & 0.999 & 1 & 0.971 & 1.030 \\
\hline Snoring & 0.010 & 0.012 & 0.377 & 1.011 & 0.987 & 1.034 \\
\hline Insomnia & 0.003 & 0.012 & 0.813 & 1.003 & 0.979 & 1.027 \\
\hline \multicolumn{7}{|l|}{$\mathrm{Pa}$} \\
\hline Tooth clenching & -0.003 & 0.023 & 0.900 & 0.997 & 0.953 & 1.044 \\
\hline Tooth grinding & 0.039 & 0.025 & 0.122 & 1.040 & 0.990 & 1.093 \\
\hline Snoring & 0.016 & 0.020 & 0.418 & 1.016 & 0.977 & 1.056 \\
\hline Insomnia & 0.028 & 0.021 & 0.188 & 1.029 & 0.986 & 1.073 \\
\hline \multicolumn{7}{|l|}{$\mathrm{Pt}$} \\
\hline Tooth clenching & 0.024 & 0.026 & 0.354 & 1.024 & 0.973 & 1.078 \\
\hline Tooth grinding & 0.008 & 0.027 & 0.756 & 1.008 & 0.956 & 1.064 \\
\hline Snoring & 0.006 & 0.022 & 0.790 & 1.006 & 0.963 & 1.050 \\
\hline Insomnia & -0.028 & 0.023 & 0.224 & 0.972 & 0.929 & 1.018 \\
\hline \multicolumn{7}{|l|}{ Sc } \\
\hline Tooth clenching & -0.011 & 0.033 & 0.729 & 0.989 & 0.926 & 1.055 \\
\hline Tooth grinding & 0.028 & 0.042 & 0.508 & 1.028 & 0.947 & 1.116 \\
\hline Snoring & -0.003 & 0.028 & 0.925 & 0.997 & 0.944 & 1.054 \\
\hline Insomnia & -0.005 & 0.030 & 0.869 & 0.995 & 0.938 & 1.056 \\
\hline \multicolumn{7}{|l|}{$\mathrm{Ma}$} \\
\hline Tooth clenching & -0.006 & 0.020 & 0.772 & 0.994 & 0.956 & 1.034 \\
\hline Tooth grinding & 0.018 & 0.021 & 0.408 & 1.018 & 0.976 & 1.062 \\
\hline Snoring & 0.001 & 0.016 & 0.969 & 1.001 & 0.969 & 1.034 \\
\hline Insomnia & 0.047 & 0.018 & $0.008^{* *}$ & 1.048 & 1.012 & 1.086 \\
\hline \multicolumn{7}{|l|}{$\mathrm{Si}$} \\
\hline Tooth clenching & 0.009 & 0.019 & 0.616 & 1.009 & 0.973 & 1.047 \\
\hline Tooth grinding & 0.015 & 0.023 & 0.523 & 1.015 & 0.970 & 1.062 \\
\hline Snoring & 0.020 & 0.016 & 0.214 & 1.020 & 0.989 & 1.053 \\
\hline Insomnia & 0.034 & 0.017 & $0.045^{*}$ & 1.034 & 1.001 & 1.069 \\
\hline
\end{tabular}

$\overline{\mathrm{SE}}$, standard error; OR, odds ratio; $\mathrm{Cl}$, confidence interval; $\mathrm{Hs}$, hypochondriasis; $\mathrm{D}$, depression; Hy, hysteria; Pd, psychopathic deviation; Mf, masculinity-femininity; Pa, paranoia; Pt, psychasthenia; Sc, schizophrenia; Ma, hypomania; Si, social introversion.

Number (\%): tooth clenching, 66 (22.1); tooth grinding, 65 (21.7); snoring, 119 (39.8); insomnia, 148 (49.5).

${ }^{*} p<0.05,{ }^{* *} p<0.01$. 
Table 3. Logistic regression analysis of RC scales on sleep disturbances $(n=299)$

\begin{tabular}{|c|c|c|c|c|c|c|}
\hline \multirow{2}{*}{ RC scales } & \multirow{2}{*}{$\beta$} & \multirow{2}{*}{ SE } & \multirow{2}{*}{$p$-value } & \multirow{2}{*}{ OR } & \multicolumn{2}{|c|}{$95 \% \mathrm{Cl}$} \\
\hline & & & & & Lower limit & Upper limit \\
\hline \multicolumn{7}{|l|}{$\mathrm{RCd}$} \\
\hline Tooth clenching & -0.035 & 0.022 & 0.117 & 0.966 & 0.924 & 1.009 \\
\hline Tooth grinding & -0.013 & 0.022 & 0.536 & 0.987 & 0.946 & 1.030 \\
\hline Snoring & 0.009 & 0.019 & 0.627 & 1.009 & 0.972 & 1.048 \\
\hline Insomnia & 0.023 & 0.020 & 0.257 & 1.023 & 0.984 & 1.064 \\
\hline \multicolumn{7}{|l|}{$\mathrm{RC} 1$} \\
\hline Tooth clenching & 0.010 & 0.019 & 0.584 & 1.010 & 0.974 & 1.049 \\
\hline Tooth grinding & -0.004 & 0.019 & 0.817 & 0.996 & 0.958 & 1.034 \\
\hline Snoring & -0.002 & 0.017 & 0.906 & 0.998 & 0.966 & 1.031 \\
\hline Insomnia & 0.040 & 0.018 & $0.029 *$ & 1.040 & 1.004 & 1.078 \\
\hline \multicolumn{7}{|l|}{$\mathrm{RC2}$} \\
\hline Tooth clenching & 0.040 & 0.021 & 0.063 & 1.040 & 0.998 & 1.085 \\
\hline Tooth grinding & 0.029 & 0.021 & 0.180 & 1.029 & 0.987 & 1.073 \\
\hline Snoring & 0.007 & 0.019 & 0.695 & 1.007 & 0.971 & 1.046 \\
\hline Insomnia & 0.013 & 0.020 & 0.527 & 1.013 & 0.974 & 1.054 \\
\hline \multicolumn{7}{|l|}{$\mathrm{RC} 3$} \\
\hline Tooth clenching & 0.002 & 0.021 & 0.910 & 1.002 & 0.962 & 1.044 \\
\hline Tooth grinding & -0.007 & 0.021 & 0.736 & 0.993 & 0.953 & 1.035 \\
\hline Snoring & -0.011 & 0.018 & 0.560 & 0.989 & 0.955 & 1.025 \\
\hline Insomnia & -0.005 & 0.019 & 0.773 & 0.995 & 0.959 & 1.032 \\
\hline \multicolumn{7}{|l|}{$\mathrm{RC4}$} \\
\hline Tooth clenching & -0.007 & 0.023 & 0.775 & 0.993 & 0.950 & 1.039 \\
\hline Tooth grinding & -0.010 & 0.023 & 0.656 & 0.990 & 0.946 & 1.036 \\
\hline Snoring & 0.038 & 0.020 & 0.059 & 1.038 & 0.999 & 1.080 \\
\hline Insomnia & 0.033 & 0.021 & 0.113 & 1.034 & 0.992 & 1.078 \\
\hline \multicolumn{7}{|l|}{$\mathrm{RC6}$} \\
\hline Tooth clenching & 0.001 & 0.021 & 0.947 & 1.001 & 0.961 & 1.043 \\
\hline Tooth grinding & 0.003 & 0.021 & 0.901 & 1.003 & 0.962 & 1.045 \\
\hline Snoring & -0.005 & 0.018 & 0.772 & 0.995 & 0.960 & 1.031 \\
\hline Insomnia & 0.005 & 0.020 & 0.821 & 1.005 & 0.965 & 1.045 \\
\hline \multicolumn{7}{|l|}{$\mathrm{RC7}$} \\
\hline Tooth clenching & 0.029 & 0.026 & 0.272 & 1.029 & 0.978 & 1.084 \\
\hline Tooth grinding & 0.043 & 0.027 & 0.106 & 1.044 & 0.991 & 1.100 \\
\hline Snoring & 0.008 & 0.023 & 0.730 & 1.008 & 0.964 & 1.054 \\
\hline Insomnia & -0.004 & 0.024 & 0.865 & 0.996 & 0.950 & 1.044 \\
\hline \multicolumn{7}{|l|}{$\mathrm{RC} 8$} \\
\hline Tooth clenching & 0.001 & 0.021 & 0.948 & 1.001 & 0.960 & 1.044 \\
\hline Tooth grinding & 0.007 & 0.021 & 0.742 & 1.007 & 0.966 & 1.050 \\
\hline Snoring & -0.013 & 0.019 & 0.493 & 0.987 & 0.952 & 1.024 \\
\hline Insomnia & 0.019 & 0.020 & 0.345 & 1.019 & 0.980 & 1.060 \\
\hline \multicolumn{7}{|l|}{$\mathrm{RC9}$} \\
\hline Tooth clenching & 0.018 & 0.028 & 0.508 & 1.018 & 0.965 & 1.075 \\
\hline Tooth grinding & 0.011 & 0.028 & 0.679 & 1.012 & 0.958 & 1.068 \\
\hline Snoring & 0.024 & 0.024 & 0.313 & 1.025 & 0.977 & 1.074 \\
\hline Insomnia & -0.009 & 0.025 & 0.721 & 0.991 & 0.943 & 1.041 \\
\hline
\end{tabular}

$\mathrm{RC}$, restructured clinical; SE, standard error; OR, odds ratio; $\mathrm{Cl}$, confidence interval; $\mathrm{RCd}$, demoralization; $\mathrm{RC1}$, somatic complaints; $\mathrm{RC2}$, low positive emotions; RC3, cynicism; RC4, antisocial behavior; RC6, ideas of persecution; RC7, dysfunctional negative emotions; RC8, aberrant experiences; RC9, hypomanic activation.

Number (\%): tooth clenching, 66 (22.1); tooth grinding, 65 (21.7); snoring, 119 (39.8); insomnia, 148 (49.5). ${ }^{*} \mathrm{p}<0.05$. 
disorder (PK), marital distress scale (MDS), hostility (Ho), overcontrolled-hostility (O-H), MacAndrew alcoholism-revised (MAC-R), addiction admission scale (AAS), addiction potential scale (APS), masculine gender role (GM), feminine gender role (GF) [15].

\section{Statistical Analysis}

Collected response data were recorded in an Excel file. All the statistical analyses were performed by IBM SPSS Statistics for Windows, Version 25.0 (IBM Co., Armonk, NY, USA). Logistic regression analyses were conducted to evaluate impacts of MMPI-2 scales on sleep disturbances. The significance level was set to $5 \%(\mathrm{p}<0.05)$.

\section{RESULTS}

The odds of tooth grinding increased significantly with the increase of $\mathrm{T}$-score of Hy scale $(\beta=0.095, \mathrm{p}=0.016)$. The risk of insomnia increased significantly as $\mathrm{T}$-score of $\mathrm{Ma}$ $(\beta=0.047, p=0.008)$ and Si scale $(\beta=0.034, p=0.045)$ increased (Table 2). The occurrence of insomnia increased significantly as T-score of RC1 ( $\beta=0.040, p=0.029)$ and PSYC scale ( $\beta=0.046, p=0.008$ ) increased (Tables 3,4$)$. The odds of tooth grinding increased significantly as T-score of ANX $(\beta=0.049, p=0.046)$ and FAM scale $(\beta=0.043, p=0.028)$ increased. The occurrence of insomnia decreased significantly with the increase of T-score of TPA scale $(\beta=-0.047$, $\mathrm{p}=0.046$ ) (Table 5). The increase of T-score of Do scale significantly contributed to the risk of tooth clenching ( $\beta=0.046, p=0.041)$. The odds of tooth grinding decreased significantly as T-score of MAC-R scale $(\beta=-0.071, p=0.003)$ increased, whereas it increased significantly as T-score of APS $(\beta=0.045, p=0.020)$ increased (Table 6). No scale of MMPI-2 significantly affected the occurrence of snoring.

Table 4. Logistic regression analysis of PSY-5 scales on sleep disturbances ( $n=299)$

\begin{tabular}{|c|c|c|c|c|c|c|}
\hline \multirow{2}{*}{ PSY-5 scales } & \multirow{2}{*}{$\beta$} & \multirow{2}{*}{ SE } & \multirow{2}{*}{$p$-value } & \multirow{2}{*}{ OR } & \multicolumn{2}{|c|}{$95 \% \mathrm{Cl}$} \\
\hline & & & & & Lower limit & Upper limit \\
\hline \multicolumn{7}{|l|}{ AGGR } \\
\hline Tooth clenching & 0.021 & 0.020 & 0.282 & 1.022 & 0.983 & 1.062 \\
\hline Tooth grinding & -0.008 & 0.020 & 0.690 & 0.992 & 0.954 & 1.032 \\
\hline Snoring & 0 & 0.017 & 0.993 & 1 & 0.967 & 1.033 \\
\hline Insomnia & -0.007 & 0.017 & 0.705 & 0.993 & 0.961 & 1.028 \\
\hline \multicolumn{7}{|l|}{ PSYC } \\
\hline Tooth clenching & 0.008 & 0.019 & 0.659 & 1.008 & 0.972 & 1.046 \\
\hline Tooth grinding & 0 & 0.019 & 0.982 & 1 & 0.962 & 1.038 \\
\hline Snoring & 0.005 & 0.016 & 0.744 & 1.005 & 0.974 & 1.038 \\
\hline Insomnia & 0.046 & 0.018 & $0.008^{* *}$ & 1.047 & 1.012 & 1.084 \\
\hline \multicolumn{7}{|l|}{ DISC } \\
\hline Tooth clenching & -0.019 & 0.019 & 0.326 & 0.981 & 0.944 & 1.019 \\
\hline Tooth grinding & 0.006 & 0.019 & 0.731 & 1.007 & 0.970 & 1.044 \\
\hline Snoring & 0.026 & 0.016 & 0.100 & 1.027 & 0.995 & 1.060 \\
\hline Insomnia & 0.012 & 0.016 & 0.451 & 1.012 & 0.981 & 1.045 \\
\hline \multicolumn{7}{|l|}{ NEGE } \\
\hline Tooth clenching & -0.001 & 0.014 & 0.931 & 0.999 & 0.971 & 1.027 \\
\hline Tooth grinding & 0.018 & 0.014 & 0.198 & 1.018 & 0.991 & 1.047 \\
\hline Snoring & 0.013 & 0.012 & 0.274 & 1.014 & 0.989 & 1.038 \\
\hline Insomnia & 0.019 & 0.013 & 0.132 & 1.019 & 0.994 & 1.045 \\
\hline \multicolumn{7}{|l|}{ INTR } \\
\hline Tooth clenching & 0.017 & 0.016 & 0.299 & 1.017 & 0.985 & 1.050 \\
\hline Tooth grinding & 0.017 & 0.016 & 0.288 & 1.017 & 0.986 & 1.050 \\
\hline Snoring & 0.010 & 0.014 & 0.481 & 1.010 & 0.982 & 1.039 \\
\hline Insomnia & 0.015 & 0.015 & 0.307 & 1.016 & 0.986 & 1.046 \\
\hline
\end{tabular}

PSY-5, personality psychopathology five; SE, standard error; OR, odds ratio; Cl, confidence interval; AGGR, aggressiveness; PSYC, psychoticism; DISC, disconstraint; NEGE, negative emotionality/neuroticism; INTR, introversion/low positive emotionality. Number (\%): tooth clenching, 66 (22.1); tooth grinding, 65 (21.7); snoring, 119 (39.8); insomnia, 148 (49.5). $* * p<0.01$. 
Table 5. Logistic regression analysis of content scales on sleep disturbances ( $n=299)$

\begin{tabular}{|c|c|c|c|c|c|c|}
\hline \multirow{2}{*}{ Content scales } & \multirow{2}{*}{$\beta$} & \multirow{2}{*}{ SE } & \multirow{2}{*}{$\mathrm{p}$-value } & \multirow{2}{*}{ OR } & \multicolumn{2}{|c|}{$95 \% \mathrm{Cl}$} \\
\hline & & & & & Lower limit & Upper limit \\
\hline \multicolumn{7}{|l|}{ ANX } \\
\hline Tooth clenching & 0.017 & 0.025 & 0.492 & 1.017 & 0.969 & 1.067 \\
\hline Tooth grinding & 0.049 & 0.024 & $0.046^{*}$ & 1.050 & 1.001 & 1.101 \\
\hline Snoring & -0.010 & 0.022 & 0.634 & 0.990 & 0.949 & 1.032 \\
\hline Insomnia & 0.038 & 0.023 & 0.101 & 1.039 & 0.993 & 1.087 \\
\hline \multicolumn{7}{|l|}{ FRS } \\
\hline Tooth clenching & 0.002 & 0.016 & 0.887 & 1.002 & 0.971 & 1.035 \\
\hline Tooth grinding & -0.007 & 0.017 & 0.666 & 0.993 & 0.960 & 1.027 \\
\hline Snoring & -0.009 & 0.014 & 0.541 & 0.991 & 0.964 & 1.019 \\
\hline Insomnia & -0.005 & 0.015 & 0.732 & 0.995 & 0.967 & 1.024 \\
\hline \multicolumn{7}{|l|}{ OBS } \\
\hline Tooth clenching & -0.006 & 0.023 & 0.785 & 0.994 & 0.949 & 1.040 \\
\hline Tooth grinding & 0.032 & 0.023 & 0.172 & 1.032 & 0.986 & 1.081 \\
\hline Snoring & 0.010 & 0.021 & 0.630 & 1.010 & 0.970 & 1.051 \\
\hline Insomnia & 0.013 & 0.021 & 0.537 & 1.013 & 0.972 & 1.056 \\
\hline \multicolumn{7}{|l|}{ DEP } \\
\hline Tooth clenching & -0.046 & 0.028 & 0.108 & 0.955 & 0.903 & 1.010 \\
\hline Tooth grinding & 0.009 & 0.027 & 0.736 & 1.009 & 0.957 & 1.064 \\
\hline Snoring & 0.015 & 0.024 & 0.540 & 1.015 & 0.968 & 1.063 \\
\hline Insomnia & 0.003 & 0.025 & 0.910 & 1.003 & 0.955 & 1.052 \\
\hline \multicolumn{7}{|l|}{ HEA } \\
\hline Tooth clenching & 0.011 & 0.022 & 0.601 & 1.011 & 0.969 & 1.056 \\
\hline Tooth grinding & -0.013 & 0.022 & 0.550 & 0.987 & 0.945 & 1.031 \\
\hline Snoring & 0.016 & 0.019 & 0.398 & 1.016 & 0.979 & 1.055 \\
\hline Insomnia & 0.033 & 0.020 & 0.102 & 1.034 & 0.993 & 1.076 \\
\hline \multicolumn{7}{|l|}{$\mathrm{BIZ}$} \\
\hline Tooth clenching & 0.015 & 0.022 & 0.502 & 1.015 & 0.972 & 1.060 \\
\hline Tooth grinding & 0.007 & 0.023 & 0.760 & 1.007 & 0.963 & 1.053 \\
\hline Snoring & -0.002 & 0.020 & 0.917 & 0.998 & 0.960 & 1.037 \\
\hline Insomnia & 0.008 & 0.020 & 0.690 & 1.008 & 0.969 & 1.049 \\
\hline \multicolumn{7}{|l|}{ ANG } \\
\hline Tooth clenching & 0.043 & 0.025 & 0.084 & 1.044 & 0.994 & 1.097 \\
\hline Tooth grinding & -0.005 & 0.026 & 0.848 & 0.995 & 0.945 & 1.047 \\
\hline Snoring & 0.022 & 0.022 & 0.317 & 1.023 & 0.979 & 1.068 \\
\hline Insomnia & 0.030 & 0.024 & 0.205 & 1.031 & 0.984 & 1.080 \\
\hline \multicolumn{7}{|l|}{ CYN } \\
\hline Tooth clenching & -0.026 & 0.032 & 0.417 & 0.975 & 0.916 & 1.037 \\
\hline Tooth grinding & -0.018 & 0.032 & 0.558 & 0.982 & 0.923 & 1.044 \\
\hline Snoring & -0.038 & 0.027 & 0.153 & 0.962 & 0.913 & 1.014 \\
\hline Insomnia & 0.004 & 0.027 & 0.879 & 1.004 & 0.952 & 1.059 \\
\hline \multicolumn{7}{|l|}{ ASP } \\
\hline Tooth clenching & -0.013 & 0.029 & 0.638 & 0.987 & 0.933 & 1.044 \\
\hline Tooth grinding & -0.004 & 0.028 & 0.875 & 0.996 & 0.942 & 1.052 \\
\hline Snoring & 0.006 & 0.024 & 0.813 & 1.006 & 0.959 & 1.055 \\
\hline Insomnia & -0.003 & 0.026 & 0.894 & 0.997 & 0.948 & 1.048 \\
\hline \multicolumn{7}{|l|}{ TPA } \\
\hline Tooth clenching & -0.013 & 0.025 & 0.597 & 0.987 & 0.939 & 1.037 \\
\hline Tooth grinding & -0.027 & 0.028 & 0.321 & 0.973 & 0.922 & 1.027 \\
\hline Snoring & 0.009 & 0.022 & 0.677 & 1.009 & 0.966 & 1.055 \\
\hline Insomnia & -0.047 & 0.024 & $0.046^{*}$ & 0.954 & 0.911 & 0.999 \\
\hline \multicolumn{7}{|l|}{ LSE } \\
\hline Tooth clenching & -0.013 & 0.028 & 0.637 & 0.987 & 0.935 & 1.042 \\
\hline Tooth grinding & 0.005 & 0.027 & 0.854 & 1.005 & 0.953 & 1.059 \\
\hline Snoring & 0.023 & 0.024 & 0.335 & 1.023 & 0.977 & 1.072 \\
\hline Insomnia & -0.006 & 0.025 & 0.798 & 0.994 & 0.947 & 1.043 \\
\hline
\end{tabular}


Table 5. Continued

\begin{tabular}{|c|c|c|c|c|c|c|}
\hline \multirow{2}{*}{ Content scales } & \multirow{2}{*}{$\beta$} & \multirow{2}{*}{ SE } & \multirow{2}{*}{ p-value } & \multirow{2}{*}{ OR } & \multicolumn{2}{|c|}{$95 \% \mathrm{Cl}$} \\
\hline & & & & & Lower limit & Upper limit \\
\hline \multicolumn{7}{|l|}{ SOD } \\
\hline Tooth clenching & 0.007 & 0.013 & 0.579 & 1.007 & 0.983 & 1.032 \\
\hline Tooth grinding & 0.003 & 0.013 & 0.815 & 1.003 & 0.978 & 1.029 \\
\hline Snoring & 0.009 & 0.011 & 0.414 & 1.009 & 0.988 & 1.030 \\
\hline Insomnia & 0.002 & 0.011 & 0.860 & 1.002 & 0.980 & 1.024 \\
\hline \multicolumn{7}{|l|}{ FAM } \\
\hline Tooth clenching & 0.010 & 0.019 & 0.621 & 1.010 & 0.972 & 1.049 \\
\hline Tooth grinding & 0.043 & 0.020 & $0.028^{*}$ & 1.044 & 1.005 & 1.086 \\
\hline Snoring & 0.024 & 0.017 & 0.168 & 1.024 & 0.990 & 1.060 \\
\hline Insomnia & 0.022 & 0.018 & 0.220 & 1.022 & 0.987 & 1.059 \\
\hline \multicolumn{7}{|l|}{ WRK } \\
\hline Tooth clenching & 0.036 & 0.033 & 0.277 & 1.037 & 0.971 & 1.107 \\
\hline Tooth grinding & -0.025 & 0.034 & 0.455 & 0.975 & 0.913 & 1.042 \\
\hline Snoring & -0.047 & 0.029 & 0.108 & 0.954 & 0.900 & 1.010 \\
\hline Insomnia & -0.008 & 0.030 & 0.799 & 0.992 & 0.935 & 1.053 \\
\hline \multicolumn{7}{|l|}{ TRT } \\
\hline Tooth clenching & -0.003 & 0.032 & 0.928 & 0.997 & 0.937 & 1.061 \\
\hline Tooth grinding & -0.037 & 0.032 & 0.249 & 0.964 & 0.905 & 1.026 \\
\hline Snoring & 0.021 & 0.027 & 0.445 & 1.021 & 0.968 & 1.077 \\
\hline Insomnia & 0.003 & 0.028 & 0.912 & 1.003 & 0.949 & 1.060 \\
\hline
\end{tabular}

SE, standard error; OR, odds ratio; $\mathrm{Cl}$, confidence interval; $\mathrm{ANX}$, anxiety; FRS, fears; OBS, obsessiveness; DEP, depression; HEA, health concerns; BIZ, bizarre mentation; ANG, anger; CYN, cynicism; ASP, antisocial practices; TPA, type A behavior; LSE, low self-esteem; SOD, social discomfort; FAM, family problems; WRK, work interference; TRT, negative treatment indicators.

Number (\%): tooth clenching, 66 (22.1); tooth grinding, 65 (21.7); snoring, 119 (39.8); insomnia, 148 (49.5).

${ }^{*} p<0.05$.

\section{DISCUSSION}

The importance of sleep in maintaining health is now becoming increasingly recognised in young adults, particularly due to our 24-hour culture of connectivity and media consumption [16,17]. The prevalence of bruxism depends on the development of civilization and the modern lifestyle [18]. The prevalence of bruxism, with either grinding or clenching was documented by $6 \%$ to $20 \%$ of adults according to epidemiologic surveys in USA student population [19]. In the current study $22.1 \%$ of subjects clenched teeth during sleep and 21.7\% ground teeth during sleep. While about one third of the United Kingdom population aged 18 and above snored at night [20], 39.8\% of subjects in the present study reported snoring. The prevalence rate of insomnia in this study was $49.5 \%$ which was higher than that noted in previous investigation in which insomnia was reported by $22.8 \%$ of the general Korean adult population [21].

There has been an increasing interest in the relationship between sleep problems and mental health. Bruxing behavior was observed in those with higher Hy scores of MMPI, indicating that there was a close relationship between Hy and bruxing behavior [22]. The mean anxiety scores of the Hospital Anxiety Depression Scales and the Hamilton Anxiety Rating Scale were statistically significant higher in patients with bruxism compared with those without bruxism. suggesting that there may be an association between bruxism and higher levels of anxiety [23]. Problems such as discord in the family are often revealed during taking the bruxer's medical history [18]. Sleep bruxism was associated positively with alcohol, caffeine, and tobacco [24]. These studies support the current result that the odds of tooth grinding increased significantly with the increase of T-score of Hy, ANX, FAM, and APS scale on MMPI-2. The present result that odds of tooth grinding decreased significantly with the increase of T-score of MAC-R scale was contrary to my expectation. This result reflects the fact that high scores on MAC-R scale may indicate persons who are socially extroverted less vulnerable to stress although high scores of MAC-R scale suggest the possibility 
Table 6. Logistic regression analysis of supplementary scales on sleep disturbances $(n=299)$

\begin{tabular}{|c|c|c|c|c|c|c|}
\hline \multirow{2}{*}{ Supplementary scales } & \multirow{2}{*}{$\beta$} & \multirow{2}{*}{ SE } & \multirow{2}{*}{$\mathrm{p}$-value } & \multirow{2}{*}{ OR } & \multicolumn{2}{|c|}{$95 \% \mathrm{Cl}$} \\
\hline & & & & & Lower limit & Upper limit \\
\hline \multicolumn{7}{|l|}{$A$} \\
\hline Tooth clenching & 0.016 & 0.041 & 0.695 & 1.016 & 0.938 & 1.100 \\
\hline Tooth grinding & -0.020 & 0.041 & 0.628 & 0.980 & 0.904 & 1.063 \\
\hline Snoring & 0.027 & 0.035 & 0.436 & 1.028 & 0.960 & 1.101 \\
\hline Insomnia & -0.034 & 0.035 & 0.342 & 0.967 & 0.902 & 1.036 \\
\hline \multicolumn{7}{|l|}{$R$} \\
\hline Tooth clenching & -0.029 & 0.023 & 0.218 & 0.972 & 0.928 & 1.017 \\
\hline Tooth grinding & -0.047 & 0.024 & 0.053 & 0.954 & 0.910 & 1.001 \\
\hline Snoring & -0.036 & 0.020 & 0.073 & 0.964 & 0.927 & 1.003 \\
\hline Insomnia & -0.022 & 0.021 & 0.297 & 0.979 & 0.939 & 1.019 \\
\hline \multicolumn{7}{|l|}{ Es } \\
\hline Tooth clenching & -0.012 & 0.027 & 0.665 & 0.988 & 0.937 & 1.043 \\
\hline Tooth grinding & -0.014 & 0.028 & 0.620 & 0.986 & 0.933 & 1.042 \\
\hline Snoring & 0.010 & 0.024 & 0.673 & 1.010 & 0.964 & 1.058 \\
\hline Insomnia & -0.006 & 0.025 & 0.793 & 0.994 & 0.947 & 1.043 \\
\hline \multicolumn{7}{|l|}{ Do } \\
\hline Tooth clenching & 0.046 & 0.023 & $0.041 *$ & 1.048 & 1.002 & 1.095 \\
\hline Tooth grinding & 0.006 & 0.023 & 0.804 & 1.006 & 0.961 & 1.052 \\
\hline Snoring & -0.011 & 0.019 & 0.577 & 0.989 & 0.953 & 1.027 \\
\hline Insomnia & 0.010 & 0.019 & 0.592 & 1.010 & 0.973 & 1.049 \\
\hline \multicolumn{7}{|l|}{$\operatorname{Re}$} \\
\hline Tooth clenching & -0.015 & 0.025 & 0.550 & 0.985 & 0.939 & 1.034 \\
\hline Tooth grinding & -0.001 & 0.025 & 0.953 & 0.999 & 0.950 & 1.049 \\
\hline Snoring & 0.006 & 0.021 & 0.774 & 1.006 & 0.966 & 1.048 \\
\hline Insomnia & -0.003 & 0.021 & 0.902 & 0.997 & 0.957 & 1.039 \\
\hline \multicolumn{7}{|l|}{ Mt } \\
\hline Tooth clenching & 0.014 & 0.035 & 0.682 & 1.014 & 0.947 & 1.087 \\
\hline Tooth grinding & -0.042 & 0.037 & 0.261 & 0.959 & 0.892 & 1.031 \\
\hline Snoring & 0.012 & 0.030 & 0.691 & 1.012 & 0.954 & 1.074 \\
\hline Insomnia & 0.057 & 0.031 & 0.066 & 1.058 & 0.996 & 1.124 \\
\hline \multicolumn{7}{|l|}{ PK } \\
\hline Tooth clenching & -0.012 & 0.039 & 0.763 & 0.988 & 0.916 & 1.067 \\
\hline Tooth grinding & 0.048 & 0.040 & 0.236 & 1.049 & 0.969 & 1.135 \\
\hline Snoring & -0.015 & 0.033 & 0.658 & 0.986 & 0.924 & 1.051 \\
\hline Insomnia & 0.031 & 0.033 & 0.348 & 1.032 & 0.966 & 1.102 \\
\hline \multicolumn{7}{|l|}{ MDS } \\
\hline Tooth clenching & 0.011 & 0.024 & 0.648 & 1.011 & 0.965 & 1.058 \\
\hline Tooth grinding & 0.040 & 0.025 & 0.108 & 1.041 & 0.991 & 1.092 \\
\hline Snoring & 0.024 & 0.021 & 0.242 & 1.025 & 0.984 & 1.067 \\
\hline Insomnia & 0.007 & 0.021 & 0.730 & 1.007 & 0.966 & 1.050 \\
\hline \multicolumn{7}{|l|}{$\mathrm{Ho}$} \\
\hline Tooth clenching & 0.005 & 0.031 & 0.876 & 1.005 & 0.946 & 1.068 \\
\hline Tooth grinding & -0.009 & 0.033 & 0.782 & 0.991 & 0.929 & 1.057 \\
\hline Snoring & -0.047 & 0.027 & 0.084 & 0.954 & 0.904 & 1.006 \\
\hline Insomnia & -0.009 & 0.027 & 0.744 & 0.991 & 0.940 & 1.046 \\
\hline \multicolumn{7}{|l|}{$\mathrm{O}-\mathrm{H}$} \\
\hline Tooth clenching & 0.013 & 0.019 & 0.493 & 1.013 & 0.976 & 1.052 \\
\hline Tooth grinding & -0.012 & 0.020 & 0.545 & 0.988 & 0.951 & 1.027 \\
\hline Snoring & -0.025 & 0.016 & 0.126 & 0.975 & 0.945 & 1.007 \\
\hline Insomnia & 0.002 & 0.017 & 0.915 & 1.002 & 0.970 & 1.035 \\
\hline \multicolumn{7}{|l|}{ MAC-R } \\
\hline Tooth clenching & -0.016 & 0.022 & 0.485 & 0.985 & 0.942 & 1.029 \\
\hline Tooth grinding & -0.071 & 0.024 & $0.003^{* *}$ & 0.931 & 0.888 & 0.977 \\
\hline Snoring & 0.007 & 0.019 & 0.711 & 1.007 & 0.970 & 1.046 \\
\hline Insomnia & 0.010 & 0.019 & 0.605 & 1.010 & 0.972 & 1.049 \\
\hline
\end{tabular}


Table 6. Continued

\begin{tabular}{|c|c|c|c|c|c|c|}
\hline \multirow{2}{*}{ Supplementary scales } & \multirow{2}{*}{$\beta$} & \multirow{2}{*}{ SE } & \multirow{2}{*}{$p$-value } & \multirow{2}{*}{ OR } & \multicolumn{2}{|c|}{$95 \% \mathrm{Cl}$} \\
\hline & & & & & Lower limit & Upper limit \\
\hline \multicolumn{7}{|l|}{ AAS } \\
\hline Tooth clenching & -0.021 & 0.023 & 0.352 & 0.979 & 0.936 & 1.024 \\
\hline Tooth grinding & -0.001 & 0.023 & 0.974 & 0.999 & 0.956 & 1.045 \\
\hline Snoring & -0.001 & 0.019 & 0.944 & 0.999 & 0.962 & 1.037 \\
\hline Insomnia & -0.011 & 0.020 & 0.589 & 0.989 & 0.951 & 1.029 \\
\hline \multicolumn{7}{|l|}{ APS } \\
\hline Tooth clenching & -0.003 & 0.019 & 0.878 & 0.997 & 0.961 & 1.034 \\
\hline Tooth grinding & 0.045 & 0.019 & $0.020 *$ & 1.046 & 1.007 & 1.087 \\
\hline Snoring & 0.005 & 0.016 & 0.742 & 1.005 & 0.975 & 1.036 \\
\hline Insomnia & -0.008 & 0.016 & 0.613 & 0.992 & 0.962 & 1.023 \\
\hline \multicolumn{7}{|l|}{ GM } \\
\hline Tooth clenching & -0.020 & 0.023 & 0.383 & 0.980 & 0.937 & 1.025 \\
\hline Tooth grinding & -0.006 & 0.024 & 0.790 & 0.994 & 0.949 & 1.041 \\
\hline Snoring & -0.001 & 0.020 & 0.977 & 0.999 & 0.961 & 1.039 \\
\hline Insomnia & -0.017 & 0.020 & 0.402 & 0.983 & 0.945 & 1.023 \\
\hline \multicolumn{7}{|l|}{ GF } \\
\hline Tooth clenching & 0.013 & 0.022 & 0.548 & 1.014 & 0.970 & 1.059 \\
\hline Tooth grinding & 0.014 & 0.023 & 0.535 & 1.014 & 0.970 & 1.061 \\
\hline Snoring & -0.033 & 0.019 & 0.081 & 0.968 & 0.933 & 1.004 \\
\hline Insomnia & -0.012 & 0.019 & 0.513 & 0.988 & 0.951 & 1.025 \\
\hline
\end{tabular}

SE, standard error; OR, odds ratio; $\mathrm{Cl}$, confidence interval; $\mathrm{A}$, anxiety; $\mathrm{R}$, repression; Es, ego strength; Do, dominance; Re, social responsibility; Mt, college maladjustment; PK, post-traumatic stress disorder; MDS, marital distress scale; Ho, hostility; O-H, overcontrolled-hostility; MAC-R, MacAndrew alcoholism-revised; AAS, addiction admission scale; APS, addiction potential scale; GM, masculine gender role; GF, feminine gender role.

Number (\%): tooth clenching, 66 (22.1); tooth grinding, 65 (21.7); snoring, 119 (39.8); insomnia, 148 (49.5).

${ }^{*} \mathrm{p}<0.05,{ }^{* *} \mathrm{p}<0.01$.

of alcohol or other substance abuse problems [15]. While high scorers on Do scale appear stronger in face-to-face personal situations and self-confident, high scorers on $\mathrm{Si}$ scale are insecure in social situations and lack self-confidence [15]. The current result that the risk of tooth clenching increased significantly with the increase of T-score of Do scale seems contradictory to the previous study [13] that the group of clenching type had higher score on Si scale.

Certain personality traits may constitute important predisposing and perpetuating factors for insomnia [25]. Mendelson et al. [26] revealed that insomniacs had higher scores on Si scale of MMPI than control subjects. SchneiderHelmert [27] concluded that patients with insomnia were somatizing and more introverted when compared to normal sleepers. Insomniacs had significantly higher MMPI scores than did normal sleepers on Hy and Sc scales, appearing to be more neurotic, anxious, and worried than their normal counterparts [28]. Shealy et al. [29] found that insomniacs who failed in treatment generally had higher scores on Ma and Sc scales of MMPI. These findings explain the present result that the odds of insomnia increased significantly with the increase of T-score of $\mathrm{Ma}, \mathrm{Si}, \mathrm{RC}$, and PSYC scale on MMPI-2. Against the expectation, the odds of insomnia decreased significantly with the increase of T-score of TPA scale in the current result, since high scorers on TPA scale are hard-driving, frequently hostile, irritable and easily annoyed [15]. Lee JS et al. suggested that there was no significant difference in T-score of TPA scale on MMPI-2 between the elderly insomniacs and noninsomniacs [30].

This study was subject to limitations comprising subjects of narrow age range, simple items of the questionnaire, and no use of polysomnography. However, It investigated all scales on MMPI-2 in contrast to previous studies focusing on clinical scales.

Conclusively, T-scores of Hy, ANX, FAM, Do, MAC-R, and APS scales on MMPI-2 affected the risk of bruxism. T-scores of Ma, Si, RC1, PSYC, and TPA scales on MMPI2 influenced the occurrence of insomnia. The psychometric 
instrument such as MMPI-2 is helpful in understanding and managing bruxism and insomnia.

\section{CONFLICT OF INTEREST}

No potential conflict of interest relevant to this article was reported.

\section{ORCID}

Hye Sook Park

https://orcid.org/0000-0002-2981-3908

\section{REFERENCES}

1. Kang JK, Lim HD, Lee YM. Association between sleep quality and psychologic factors among university students in Korea. Korean J Oral Med 2008;33:257-267.

2. Jang SM, Jeon HM, Kim KH, et al. Application of botulinum toxin on masticatory muscle of patients with bruxism. J Oral Med Pain 2014;39:55-62.

3. Hui DS, Chan JK, Ho AS, Choy DK, Lai CK, Leung RC. Prevalence of snoring and sleep-disordered breathing in a student population. Chest 1999;116:1530-1536.

4. Kim KS. Factors contributing to temporomandibular disorders. J Korean Dent Assoc 1991;29:354-358.

5. Ahlberg J, Lobbezoo F, Ahlberg K, et al. Self-reported bruxism mirrors anxiety and stress in adults. Med Oral Patol Oral Cir Bucal 2013;18:e7-e11.

6. Li AM, Au CT, So HK, Lau J, Ng PC, Wing YK. Prevalence and risk factors of habitual snoring in primary school children. Chest 2010;138:519-527.

7. Vgontzas AN. Vgontzas. The diagnosis and treatment of chronic insomnia in adults. Sleep 2005;28:1047-1048.

8. Manfredini D, Landi N, Fantoni F, Segù M, Bosco M. Anxiety symptoms in clinically diagnosed bruxers. J Oral Rehabil 2005;32:584-588.

9. Restrepo CC, Vásquez LM, Alvarez M, Valencia I. Personality traits and temporomandibular disorders in a group of children with bruxing behaviour. J Oral Rehabil 2008;35:585-593.

10. Enright PL, Newman AB, Wahl PW, Manolio TA, Haponik EF, Boyle PJ. Prevalence and correlates of snoring and observed apneas in 5,201 older adults. Sleep 1996;19:531-538.

11. Buysse DJ, Angst J, Gamma A, Ajdacic V, Eich D, Rössler W. Prevalence, course, and comorbidity of insomnia and depression in young adults. Sleep 2008;31:473-480.

12. Singareddy R, Vgontzas AN, Fernandez-Mendoza J, et al. Risk factors for incident chronic insomnia: a general population prospective study. Sleep Med 2012;13:346-353.

13. Park BO, Lee HC, Jang MJ, Shim JC. MMPI profiles of the patients with bruxism. J Korean Neuropsychiatr Assoc 1999;38:523-529.

14. Aikens JE, Caruana-Montaldo B, Vanable PA, Tadimeti L, Mendelson WB. MMPI correlates of sleep and respiratory disturbance in obstructive sleep apnea. Sleep 1999;22:362-369.

15. Graham JR. MMPI-2: assessing personality and psychopathology. 5th ed. New York: Oxford University Press; 2011. pp. 63-221.

16. Cain N, Gradisar M. Electronic media use and sleep in schoolaged children and adolescents: a review. Sleep Med 2010;11:735742 .

17. Lemola S, Perkinson-Gloor N, Brand S, Dewald-Kaufmann JF, Grob A. Adolescents' electronic media use at night, sleep disturbance, and depressive symptoms in the smartphone age. J Youth Adolesc 2015;44:405-418.

18. Wieckiewicz M, Paradowska-Stolarz A, Wieckiewicz W. Psychosocial aspects of bruxism: the most paramount factor influencing teeth grinding. Biomed Res Int 2014;2014: 469187.

19. Glaros AG. Incidence of diurnal and nocturnal bruxism. J Prosthet Dent 1981;45:545-549.

20. Lechner M, Breeze CE, Ohayon MM, Kotecha B. Snoring and breathing pauses during sleep: interview survey of a United Kingdom population sample reveals a significant increase in the rates of sleep apnoea and obesity over the last 20 years - data from the UK sleep survey. Sleep Med 2019;54:250-256.

21. Cho YW, Shin WC, Yun CH, Hong SB, Kim J, Earley CJ. Epidemiology of insomnia in korean adults: prevalence and associated factors. J Clin Neurol 2009;5:20-23.

22. Molina OF, Peixoto MGS, Santos ZC, Penoni JDS, Aquilino RN, Peixoto MAS. Bruxism as mechanism subserving hysteria: a new theory. Rev Neurocienc 2008;16:262-268.

23. Gungormus Z, Erciyas K. Evaluation of the relationship between anxiety and depression and bruxism. J Int Med Res 2009;37:547550.

24. Bertazzo-Silveira E, Kruger CM, Porto De Toledo I, et al. Association between sleep bruxism and alcohol, caffeine, tobacco, and drug abuse: a systematic review. J Am Dent Assoc 2016;147:859866.

25. van de Laar M, Verbeek I, Pevernagie D, Aldenkamp A, Overeem $\mathrm{S}$. The role of personality traits in insomnia. Sleep Med Rev 2010;14:61-68.

26. Mendelson WB, Garnett D, Gillin JC, Weingartner H. The experience of insomnia and daytime and nighttime functioning. Psychiatry Res 1984;12:235-250.

27. Schneider-Helmert D. Twenty-four-hour sleep-wake function and personality patterns in chronic insomniacs and healthy controls. Sleep 1987;10:452-462.

28. Freedman RR, Sattler HL. Physiological and psychological factors in sleep-onset insomnia. J Abnorm Psychol 1982;91:380-389.

29. Shealy RC, Lowe JD, Ritzler BA. Sleep onset insomnia: personality characteristics and treatment outcome. J Consult Clin Psychol 1980;48: 659-661.

30. Lee JS, Yoo RY, Nam HJ, et al. Clinical characteristics of elderly patients aged over 65 years with insomnia symptoms. J Korean Sleep Res Soc 2013;10:25-30. 
Appendix 1. Form and contents of questionnaire

\section{QUESTIONNAIRE}

Name of College :

Name of Department :

Grade :

Name :

Gender :

Please answer the following questions by checking the one answer which describes you.

1. Have you ever clenched your teeth during sleep or awaked with your teeth clenched?

1) Yes

2) No

2. Have you ever ground your teeth during sleep or heard that you were a bruxer?
1) Yes
2) No

3. Have you ever snored during sleep or heard that you were a snorer?

$\begin{array}{ll}\text { 1) Yes } & \text { 2) No }\end{array}$

4. Have you difficulty initiating or maintaining sleep at night or nonrestorative sleep?

$\begin{array}{ll}\text { 1) Yes } & \text { 2) No }\end{array}$

Thank you for your cooperation. 